\title{
Corela
}

Cognition, représentation, langage

HS-1 1 | 2012

RJC Cotexte, contexte, situation

\section{Genèse de l'évolution des représentations de la communication exolingue par des enfants plurilingues : au commencement était l'apprentissage de la langue étrangère.}

\section{Emmanuelle Le Pichon - Vorstman}

\section{(2) OpenEdition}

Journals

Édition électronique

URL : http://journals.openedition.org/corela/2620

DOI : $10.4000 /$ corela.2620

ISSN : 1638-573X

Éditeur

Cercle linguistique du Centre et de l'Ouest - CerLICO

Référence électronique

Emmanuelle Le Pichon - Vorstman, « Genèse de l'évolution des représentations de la communication exolingue par des enfants plurilingues : au commencement était l'apprentissage de la langue

étrangère. ", Corela [En ligne], HS-11 | 2012, mis en ligne le 03 février 2012, consulté le 02 mai 2019

URL : http://journals.openedition.org/corela/2620 ; DOI : 10.4000/corela.2620

Ce document a été généré automatiquement le 2 mai 2019.

\section{(i) (ㅇ)}

Corela - cognition, représentation, langage est mis à disposition selon les termes de la licence Creative Commons Attribution - Pas d'Utilisation Commerciale - Partage dans les Mêmes Conditions 4.0 International. 


\title{
Genèse de l'évolution des
}

représentations de la communication exolingue par des enfants plurilingues: au commencement était l'apprentissage de la langue étrangère.

\author{
Emmanuelle Le Pichon - Vorstman
}

\section{Introduction}

1 L'étude du comportement communicatif d'enfants bilingues simultanés à montré que, très jeunes, les enfants bilingues étaient capable de sélectionner l'une des langues de leur répertoire en fonction du répertoire supposé de leur interlocuteur (Cenoz \& Jessner, 2000 : 48, Genesee, Tucker \& Lambert, 1975 ; Jessner, 1997 ; Jorda, 2005 ; Hoffmann, 2001, De Houwer, 1995). Peut-on alors en conclure que l'acquisition de deux langues en bas âge favorise positivement la compétence de communication des enfants bilingues par rapport à d'autres types de plurilinguismes?

2 Selon Coste, Moore et Zarate, 1997, le terme plurilingue renvoie à un contenu flexible de compétences qui s'oppose au concept de bilinguisme simultané ou consécutif évoqué plus haut (Coste, Moore \& Zarate, 1997 :12). De ce fait, dans le présent article, les enfants ayant été exposés à plus d'une langue au cours de leur développement sont tous considérés comme étant plurilingues même si ils sont monolingues en apprentissage d'une langue étrangère, c'est-à-dire même si leur compétence en langue étrangère/ seconde est encore très limitée.

On peut alors formuler autrement la question posée et se demander si le bilinguisme précoce, compris comme l'exposition à plus d'une langue à un très jeune âge et quotidiennement, permettrait de développer des représentations de la situation de 
communication plus positives que l'apprentissage d'une langue étrangère ou seconde à un âge plus tardif.

4 Un certain nombre de recherches relativement récentes semblent remettre en cause une réponse peut-être hâtive à cette question. En effet, Rubin et Turner, par exemple, ont observé de meilleures performances métalinguistiques chez les enfants exposés à l'apprentissage intensif d'une langue lors d'une scolarisation récente en milieu immersif par rapport aux performances d'enfants bilingues scolarisés en milieu monolingue (Rubin \& Turner, 1989). D'autres comme Campbell et Sais (1995), Yelland, Pollard et Mercuri (1993), Bruck et Genesee (1995) ont observé la disparition des avantages métalinguistiques observés chez les enfants bilingues à la fin du cours préparatoire.

5 Suite à l'impossibilité d'identifier formellement des différences significatives entre les groupes d'enfants monolingues et bilingues, certains chercheurs ont avancé l'idée selon laquelle d'autres facteurs pourraient avoir une influence plus importance sur la conscience métacognitive de l'enfant que le bilinguisme en tant que tel (Bialystok, 2001; pour une discussion, voir Genesee \& Nicoladis, 1997 ; Grosjean, 1997).

\section{Conscience métacognitive}

6 Le terme «métacognition » a été employé dans de nombreuses études concernant la compréhension, la communication, le langage, la perception, l'attention et/ou la résolution de problème (Flavell, 2004). Selon Flavell, la résolution d'un problème implique une aptitude à contrôler la difficulté de la tâche désignée. Selon lui, l'adaptation de la connaissance métacognitive, (ou conscience métacognitive), à la résolution de la tâche inclut deux éléments essentiels : l'anticipation de la tâche et une évaluation de son issue.

7 En effet, dans les définitions diverses de la métacognition apparaissent de manière récurrente les notions de connaissance, conscience, et contrôle des stratégies (voir Brown, Bransford, Ferrara \& Campione, 1983 ; Baird, 1990 ; Lee Swanson, 1990; Biggs \& Moore, 1993).

\section{Stratégie}

8 La notion de stratégies est, en effet, centrale dans le concept de métacognition qui inclut mémoire, compréhension, apprentissage et attention (voir par exemple Brown \& Day, 1983, Beacco, 2007). Oxford définie une stratégie comme étant « un plan conscient dont l'élaboration vise à atteindre un objectif » (Oxford, 2003 : 274). Elle souligne, elle aussi, l'importance des notions de conscience, contrôle, objectif et intention. Pour Goldstein and Levin, la résolution de problème ne peut avoir lieu que lorsqu'un objectif ne peut être atteint de manière automatique, donc exigeant le recours à la compétence stratégique (Goldstein \& Levin, 1987). Pour Zeidner and Matthews, la compétence stratégique constitue une part essentielle des fonctions exécutives inclues dans la résolution de problème (Zeidner \& Matthews, 2000 : 598).

9 Ainsi, la compétence stratégique est définie dans l'étude présente comme étant représenté par la conscience du sujet de l'existence de certaines stratégies de communication et par son intention d'employer ces stratégies pour communiquer. 


\section{Définitions des stratégies de communication}

10 Faerch et Kasper ont décrit les stratégies de communication comme 'des plans potentiellement conscients pour résoudre ce qui peut se présenter à un individu comme problématique pour atteindre un objectif communicatif particulier (Faerch \& Kasper, 1983 : 23). Ainsi, dans cet article, l'aptitude des enfants à évoquer ces stratégies et leur intention de les employer constituent la compétence stratégique. Ces deux éléments (intention de communiquer (Dörnyei \& Scott, 1997 : 179) et stratégies rapportées par les enfants) sont considérés comme des éléments essentiels permettant à la participation à une éventuelle interaction d'avoir lieu lors d'un échange (MacIntyre, Clément, Dörnyei, \& Noel, 1998). Dans la définition de Faerch et Kasper, la situation de communication exolingue est donc considérée comme intrinsèque à l'élaboration d'un plan stratégique.

\section{La situation de communication exolingue}

11 En principe, depuis les recherches de Krashen (1981) l'exposition à deux langues avant l'entrée à l'école est considérée comme un processus acquisitionnel. Ce processus est souvent opposé à celui d'apprentissage. L'apprentissage d'une langue seconde ou étrangère (respectivement abrégées, L2 ou LE), est associé à un âge plus avancé et à un contexte formel d'exposition à la langue nouvelle, c'est-à-dire le plus souvent l'école. L'un des arguments essentiels de cette dichotomie serait que le degré de conscience impliqué dans le processus varie en fonction de l'âge et du contexte d'exposition à la nouvelle langue. La situation exolingue est intrinsèque à l'acquisition/ apprentissage des langues étrangères.

12 Terme introduit par Porquier en 1979, repris et explicité par la suite en particulier par Alber et Py en 1985, cette situation met en scène un déséquilibre concernant les savoirs linguistiques respectifs des participants à l'interaction. Elle est caractérisée par un écart dans les performances des sujets qui interagissent dans la langue en question, et peut se caractériser par une différence d'accent ou dans le cas d'une situation exolingue extrême, par le fait que les participants n'ont aucune langue en commun. La situation de communication exolingue extrême, pour être menée à bien, nécessite de considérer tous les indices de contextualisation à la disposition des interactants dans le contexte imposé par cette situation. Du fait de la radicalité de cette situation, les indices devraient devenir évidents puisque le contenu sémantique des mots ne peut être pris en compte. Le terme 'indices de contextualisation' a été proposé par Gumperz pour désigner des éléments linguistiques, paralinguistiques ou kinésiques qui signalent aux interactants une interprétation possible de la situation de communication, par exemple l'intonation, les mimiques, les gestes, ou les regards (Cosnier, 1991: 13 ; Gumperz, 1982 :145). On comprend qu'elle suppose avant tout une volonté de coopérer (identifié par MacIntyre et al. (1998) "Willingness To Communicate ») et une fois cette volonté de communiquer exprimée, la mobilisation de stratégies pour mener à bien l'interaction (le Pichon et al. sous presse).

13 Si les recherches récentes montrent que le jeune enfant exposé à plus d'une langue découvre très jeune l'existence de systèmes sémiotiques distincts (De Houwer, 1995), plus le petit enfant grandit et plus il est amené à prendre conscience de cette situation de déséquilibre linguistique étant de plus en plus souvent amené à quitter ses parents et à 
être confronté à des situations d'interactions exolingues, par exemple, lors de son entrée à l'école. En d'autres termes, plus l'apprentissage de la langue cible est tardif et plus l'enfant risque de mesurer l'ampleur de la situation d'exolinguisme. Ce que l'on ne sait pas encore, c'est dans quelle mesure cette situation influence le développement métacognitif de l'enfant. Cette situation influence-t-elle son désir de communiquer et si oui, de quelle manière?

\section{Constructions cognitives et événements liés aux biographies langagières}

14 Les recherches sur la conscience métacognitive des jeunes enfants plurilingues, et plus particulièrement en terme de compétence stratégique, sont à notre connaissance très rares (Chamot \& El'Dinary, 1999; Benson, 2009). Les premières années de la vie des enfants plurilingues, comme celles des enfants monolingues, sont marquées par une constante interaction entre développement cognitif et langagier (Noyau, 1998). Les enfants construisent des notions qu'ils expriment à travers les mots de leur(s) langue(s) fondées sur leurs expériences et dépendant de leur développement cognitif. Ces interactions constituent des événements linguistiques qui jalonnent leur vie et qui ne peuvent être dissociés des interactions au cours desquelles ils apparaissent, l'interaction étant le canal indispensable d'acquisition du langage en général et des langues en particulier.

Pour les enfants plurilingues ou en passe de le devenir, ces événements linguistiques peuvent être constitués par un déménagement, l'entrée à l'école ou de simples vacances dans le pays d'origine des parents. Certains chercheurs (Bialystok, 2009) déplorent le fait que ces facteurs d'ordre socioculturels soient si rarement pris en compte dans les recherches de grande ampleur sur le bilinguisme au sens large du terme, soit la capacité de comprendre et de s'exprimer dans plus d'une langue (voir Myers-Scotton, 2006; Pavlenko, 2004). La prise en compte des contextes d'acquisition/ apprentissage dans les recherches sur le plurilinguisme précoce se limite souvent à l'analyse des effets de l'âge d'apprentissage de la langue étrangère en contexte scolaire, ou bien encore consiste à comparer différentes méthodes d'apprentissage et leur influence sur le développement (méta)cognitif des enfants plurilingues (pour une discussion voir, Le Pichon, Vorstman, de Swart, van den Bergh, 2009 ; Bialystok, 2009 ; Mondada \& Pekarek, 2005 ; Cole, 1995). De Houwer (1995), pour résoudre ce problème de l'hétérogénéité des répertoires plurilingues, propose une définition très restreinte du bilinguisme en tant qu'exposition à deux langues durant les premiers mois de la vie. Pourtant, force est de constater que la difficulté de la prise en compte de l'hétérogénéité de ces facteurs contextuels d'appropriation de l'objet langue dans les recherches sur le plurilinguisme explique le fait qu'elles se fassent rares (Mondada \& Pekarek, 2005 ; Bialystok, 2009 ; Porquier \& Py, 2004 ; Cummins, 1979).

16 Considérer le plurilinguisme comme un processus développemental implique la prise en compte de l'interaction d'événements linguistiques et de processus cognitifs dans des contextes d'acquisition/ apprentissage variés. Deux facteurs semblent particulièrement prégnants (Narcy-Combes, 2008) : (1) Le contexte de l'acquisition de chacune des langues et (2) l'âge des enfants au moment du premier contact avec chacune des langues. 
17 Envisager des 'contextes', c'est réhabiliter des contextes éducationnels de formation qui interagissent et influencent le développement métacognitif de l'enfant. D'un point de vue empirique, Porquier et Py posent alors la question de savoir comment 'rassembler dans une même approche théorique des constructions cognitives et des événements biographiques ?' (2004). C'est la question à laquelle nous avons tenté de répondre avec la présente recherche.

\section{L'approche par les biographies langagières}

18 Ayant défini le processus d'apprentissage en tant qu'activité indissociable du contexte dans lequel il se déroule, et situé la notion d'apprentissage comme essentielle du point de vue du développement métacognitif de l'enfant, on peut du même coup, envisager d'y associer une approche par les biographies langagières, attestée par de plus en plus de chercheurs dans différents domaines de la linguistique (Castellotti, 2008). Une telle approche permettrait de spécifier certains des apports de l'apprentissage scolaire d'une L2 par contraste avec d'autres types d'apprentissages situés dans d'autres contextes situationnels.

19 Les enfants, les enseignants et les familles qui fréquentent les écoles internationales, forment une communauté au sens de groupe social, dont l'une des caractéristiques est l'hétérogénéité des répertoires linguistiques de ses membres. Elle trouve, presque de façon contradictoire, une certaine homogénéité dans l'hétérogénéité des répertoires linguistiques des ses membres. Le contexte des écoles internationales est alors caractérisé par l'omniprésence de contacts de langues, espace par excellence de diversités linguistique et culturelle. Les situations de communication exolingue créent ce que l'on pourrait appeler des contextes sociaux situationnels où l'on tente de valoriser la diversité des ressources linguistiques et culturelles des enfants. Dans l'une des écoles visitées par exemple, en dernière année de maternelle, chaque enfant à son tour, est la vedette de l'école. Il ou elle prépare un poster qui le/la caractérise sur lequel se côtoient photos de la famille, activités, dessins, décorations, drapeaux et qui sera affiché dans le couloir pendant toute une semaine bien en vue de chacun. En conséquence, malgré le choix d'une ou deux langues d'instruction, les enseignants puisent dans les répertoires plurilingues des enfants comme ils en témoignent eux-mêmes.

$\mathrm{Du}$ point de vue du développement de la compétence dite plurilingue, ce contexte institutionnel semble donc idéal. Les écoles 'internationales' permettraient alors, par la prise en compte de ces contacts de langue concrètement réinvestis à l'intérieur de la classe, 'de se reconnaître et de s'affirmer en tant qu'acteur social plurilingue' (Moore \& Castellotti, 2008). De ce postulat naissent trois questions qui sous-tendent la présente recherche :

1. Ce contexte de valorisations linguistique et culturelle suffit-il au développement optimal de la compétence plurilingue?

2. Quels types d'acquisition peuvent être observés en fonction du type d'acquisition/ apprentissage de la langue étrangère ?

3. Les enfants pour l'éducation desquels les conditions institutionnelles "optimales » sont réunies ont-ils tous le même avantage face à la situation de communication exolingue ? 


\section{Objectifs et hypothèse}

21 La présente recherche a pour objectif d'évaluer l'apport de l'apprentissage d'une langue nouvelle en contexte scolaire sur les représentations métacommunicationnelles des enfants plurilingues par rapport au bilinguisme précoce.

Suivant l'hypothèse selon laquelle le contexte d'acquisition de chaque langue pourrait jouer un rôle central dans le développement de la compétence plurilingue, nous proposons que certains aspects de la compétence communicative des enfants plurilingues tels que les représentations qu'ils se font de la situation de communication exolingue et les stratégies qu'ils envisagent d'employer, dépendent plus de l'expérience d'apprentissage de la langue étrangère en contexte formel que de l'acquisition simultanée de deux langues en milieu naturel.

Il nous a semblé que l'exposition à une langue nouvelle en contexte formel (éducationnel) et après l'âge de 4 ans pourrait générer chez les enfants une expérience d'apprentissage consciente que ne génèrerait pas forcément l'acquisition de deux langues en milieu naturel et avant l'âge de quatre ans. Nous avons alors émis l'hypothèse selon laquelle cette conscience, qui nous semble être générée par l'apprentissage d'une langue nouvelle, peut affecter l'intentionnalité des enfants à envisager l'interaction et leur accès aux stratégies communicatives.

Cette expérience est appelée dans notre étude Expérience d'Apprentissage d'une Langue Étrangère, abrégée LLE (de l'Anglais, Language Learning Experience, voir Le Pichon et al., 2009).

\section{Méthode}

\section{Population}

La présente recherche prend place dans le cadre de trois écoles internationales aux PaysBas, aux Etats-Unis d'Amérique et en Suisse, deux pays et un canton, non-francophones. Une série de 101 entretiens individuels auprès d'enfants âgés de 4,1/2 à 11 ans scolarisés dans trois écoles (Françaises) internationales (Pays-Bas, États-Unis, Suisse) a été menée au sein de trois établissements scolaires. Aux Pays-Bas, nous avons rencontré 28 enfants âgés de 7 à 11 ans (âge moyen= 9,4), en Suisse, 19 enfants âgés de 5 à 6 ans (âge moyen= 5,5), et aux États-Unis, 54 enfants âgés de 4,5 à 6 ans (âge moyen= 5,5). Dans chacune des classes, tous les enfants ont participé aux entretiens.

Comme évoqué précédemment, la situation exolingue est intrinsèque à l'acquisition/ apprentissage des langues étrangères. L'apprenant d'une langue est quotidiennement confronté à des obstacles relatifs à la langue elle-même. Manquant d'éléments linguistiques pour s'exprimer (tout à fait librement) dans la langue de l'interaction, l'apprenant doit avoir recours à des stratégies communicatives pour mener à bien l'interaction. Sans ces stratégies, l'interaction risque d'être interrompue. Il est nécessaire pour résoudre un problème lié à la situation de communication exolingue de faire preuve à la fois d'une volonté forte de communication et de mettre en place ces stratégies communicatives. 
Tous les enfants inclus dans la présente recherche sont en contact plus ou moins permanent avec des situations exolingues. S'ils ne sont pas apprenants d'une langue euxmêmes, ils sont tous en contact dans la classe avec des enfants qui apprennent l'une des langues de l'école (le français le plus souvent, mais aussi l'anglais, l'allemand ou le néerlandais suivant les écoles).

Tableau 1. Caractéristiques démographiques des participants

\begin{tabular}{|l|l|l|l|l|}
\hline & N & Âge moyen (4-11) & SD & Sexe \\
\hline LLE & 72 & 6,9 & 2.0 & $47 \%$ filles \\
\hline nLLE & 29 & 6,07 & 1.6 & $45 \%$ filles \\
\hline LLE+ nLLE & 101 & 6,7 & & $46 \%$ filles \\
\hline
\end{tabular}

Au total, 101 enfants âgés de 4 à 11 ans ont participé aux interviews. Sur ce tableau sont présentés l'âge moyen et la répartition des sexes des enfants selon les groupes LLE versus nLLE et sur la totalité du groupe (voir l'explication de la répartition des groupes ci-dessous).

\section{Procédure}

Afin de pouvoir observer l'attitude des enfants face à ces situations et les stratégies utilisées pour identifier, qualifier et éventuellement résoudre ces situations, les participants ont été confrontés à des tâches relevant de situations de communication exolingues extrêmes. Ces situations sont caractérisées par le fait que les interlocuteurs ne disposent pas de ressources linguistiques communes. Ils ne parlent pas la ou les mêmes langues. La résolution de ces situations nécessite alors la mobilisation de principes communs à toute interaction verbale tels que paralinguistiques (geste, mimique, intonations). Ces stratégies permettent de pallier l'absence de mots pour transmettre des informations.

Les entretiens individuels et semi-ouverts duraient au maximum 13 minutes en raison du jeune âge des enfants. Chaque entretien se terminait par la question suivante, question analysée dans la présente étude (pour une analyse des autres tâches incluses dans l'entretien voir Le Pichon, De Swart, Ceginskas et van den Bergh, 2009) :

'Tu es dans un parc et tu veux jouer avec un(e) enfant qui ne parle pas ou qui ne comprend pas les mêmes langues que toi. Qu'est -ce que tu vas faire?'

Les interventions des enfants étaient valorisées indépendamment des formes produites ou de la pertinence des stratégies énoncées par une expression d'appréciation à connotation fortement positive et au caractère emphatique du type: 'bravo', 'quelle bonne idée !', 'formidable', 'bon travail !'.

\section{LLE versus nLLE}

De la prise en compte des parcours linguistiques des enfants résulte une classification en deux groupes distincts.

1. Le premier groupe, appelé LLE, (abrégé de l'anglais 'Language Learning Experience' en français Experience d'Apprentissage d'une Langue) réuni les enfants avec expérience d'apprentissage 
d'une langue étrangère en milieu formel après l'âge de 4 ans. Il est constitué d'enfants dits 'monolingues', (c'est-à-dire dont le développement linguistique de la langue cible n'est pas suffisant pour pouvoir s'exprimer en langue cible sur des sujets simples) ou d'enfants dits 'bilingues' qui après l'âge de 4 ans ont appris ou sont en cours d'apprentissage d'une langue étrangère à l'école.

2. Le deuxième groupe, appelé nLLE (abrégé de l'anglais 'non Language Learning Experience' en français 'sans Expérience d'Apprentissage de la Langue Étrangère), réuni les enfants sans une expérience explicite d'apprentissage d'une langue étrangère en milieu formel à partir de l'âge de quatre ans. Il est constitué des enfants qui avant l'âge de 4 ans ont été en contact significatif avec une langue supplémentaire, (parents, crèches...) soit, des enfants dits 'bilingues' mais sans expérience explicite de l'apprentissage d'une nouvelle langue étrangère en milieu formel.

Les entretiens avec les enfants et avec leurs enseignantes ont apporté les renseignements concernant les âges d'exposition à chacune des langues en fonction des environnements socioculturels variés. Plus précisément, ces détails nous renseignent en termes de connaissances préalables des langues, de langues maternelles, d'attitudes face à la langue cible, d'expériences d'apprentissage. Les informations issues de ces entretiens nous ont permis de classer les biographies langagières des enfants comme indiqué dans le tableau ci-dessous dans la colonne « répertoires linguistiques ».

\section{Classification des données}

La classification et l'analyse des réponses des participants ont été guidées par les questions suivantes :

1. Est-ce que les enfants ont l'intention de communiquer? C'est-à-dire qu'elle est leur disposition envers la coopération dans cette situation imaginaire à l'issue de la tâche?

2. Comment envisagent-ils de mener l'interaction? C'est-à-dire, quelles sont les stratégies auxquelles les enfants vont penser pour mener à bien ces interactions?

Ci-dessous des exemples de réponses sont donnés pour comprendre la démarche :

Tableau 2 : Exemples de réponses des enfants

\begin{tabular}{|l|l|l|l|l|}
\hline Prénoms & Ages & $\begin{array}{l}\text { Répertoires } \\
\text { linguistiques }\end{array}$ & $\begin{array}{l}\text { LLE } \\
\text { versus } \\
\text { nLLE }\end{array}$ & Réponses \\
\hline $\begin{array}{l}\text { Isabella } \\
\text { 2. Elisa }\end{array}$ & $\begin{array}{l}6 \\
\text { ans }\end{array}$ & $\begin{array}{l}\text { Bilingue Anglais et } \\
\text { Cantonais. } \\
\text { apprend le Français à } \\
\text { l'école }\end{array}$ & $\begin{array}{l}\text { Anglais. Elle apprend le } \\
\text { Français à l'école }\end{array}$ & $\begin{array}{l}\text { LLE } \\
\text { Iingnes. }\end{array}$ \\
\hline 3. Mike & $\begin{array}{l}6 \\
\text { ans }\end{array}$ & $\begin{array}{l}\text { Monolingue Anglais, } \\
\text { en apprentissage du } \\
\text { Français à l'école }\end{array}$ & LLE & $\begin{array}{l}\text { I try to learn what he is saying. When I get } \\
\text { the hang of it, I speak just like him. }\end{array}$ \\
\hline
\end{tabular}




\begin{tabular}{|c|c|c|c|c|}
\hline 4. Maria & $\begin{array}{l}6 \\
\text { ans }\end{array}$ & $\begin{array}{lr}\text { Bilingue, } & \text { Anglais, } \\
\text { Espagnol } & \text { en } \\
\text { apprentissage } & \text { du } \\
\text { Français à l'école } & \end{array}$ & LLE & $\begin{array}{l}\text { Je sais pas quoi lui dire. Je viens vers elle. } \\
\text { J'essaie de la faire jouer avec moi. Je lui } \\
\text { montre ce que je fais et je lui fais signe de } \\
\text { me suivre. }\end{array}$ \\
\hline 5. Emma & $\begin{array}{l}6 \\
\text { ans }\end{array}$ & $\begin{array}{l}\text { Bilingue Anglais } \\
\text { Français }\end{array}$ & nLLE & $\begin{array}{l}\text { Je suis pas contente. Je vais pas jouer parce } \\
\text { que je comprends pas la langue. }\end{array}$ \\
\hline 6. Samuel & $\begin{array}{l}6 \\
\text { ans }\end{array}$ & $\begin{array}{l}\text { Monolingue Anglais. } \\
\text { En apprentissage du } \\
\text { Français à l'école }\end{array}$ & LLE & $\begin{array}{l}\text { Je sais pas. If he doesn' } t \text { speak English or } \\
\text { French, I couldn't ask him. I could just play } \\
\text { with him. }\end{array}$ \\
\hline 7. Bryan & $\begin{array}{l}6 \\
\text { ans }\end{array}$ & $\begin{array}{l}\text { Bilingue } \quad \text { Anglais } \\
\text { Français }\end{array}$ & nLLE & $\begin{array}{l}\text { Trouver une autre personne; jouer à la } \\
\text { maison. }\end{array}$ \\
\hline 8. Aurora & $\begin{array}{l}6 \\
\text { ans }\end{array}$ & $\begin{array}{l}\text { Monolingue (Anglais) } \\
\text { En apprentissage du } \\
\text { Français à l'école. }\end{array}$ & LLE & $\begin{array}{l}\text { I'm gonna get my slate board and write it } \\
\text { down. }\end{array}$ \\
\hline 9. Jacob & $\begin{array}{l}6 \\
\text { ans }\end{array}$ & $\begin{array}{l}\text { Monolingue (Anglais) } \\
\text { en apprentissage du } \\
\text { français à l'école. }\end{array}$ & LLE & I just show what to do! \\
\hline 10. Louis & $\begin{array}{l}6 \\
\text { ans }\end{array}$ & $\begin{array}{ll}\text { Bilingue Anglais et } \\
\text { Français, } & \text { en } \\
\text { apprentissage } & \text { de } \\
\text { l'allemand } & \end{array}$ & LLE & $\begin{array}{l}\text { Parler comme Deutsch. Je parle une autre } \\
\text { langue. Je vais parler moitié suisse. Etre } \\
\text { gentil, l'aider, jouer si il est triste avec lui }\end{array}$ \\
\hline 11. Rob & $\begin{array}{l}6 \\
\text { ans }\end{array}$ & $\begin{array}{l}\text { Monolingue } \\
\text { apprentissage } \\
\text { l'Allemand }\end{array}$ & LLE & $\begin{array}{l}\text { Je vais lui demander quelle langue il parle } \\
\text { en français. Je lui dis les mots que je } \\
\text { connais. Je demande à ma mère. J'espère } \\
\text { qu'il comprend. Je lui dis: bouge pas! Je } \\
\text { fais le langage des signes. }\end{array}$ \\
\hline 12. Alicia & $\begin{array}{l}5 \\
\text { ans }\end{array}$ & $\begin{array}{l}\text { Bilingue, Français et } \\
\text { Anglais }\end{array}$ & nLLE & $\begin{array}{l}\text { She speaks Italian? She speaks anything? } \\
\text { French? I don't know. I do nothing. I don't } \\
\text { play with her. }\end{array}$ \\
\hline
\end{tabular}

\section{Description des critères pour classer les stratégies}

Pour chaque réponse des critères ont été définis pour classer les stratégies utilisées par les enfants. Toute démarche allant dans le sens de la résolution du problème posé par la communication est considérée comme stratégique qu'elle soit pertinente ou non. Ainsi, les processus intentionnels engagés pour contrôler l'échange imaginaire sont tous considérés comme étant une indication de la présence de la compétence stratégique de l'enfant. Le niveau de compétence stratégique de chaque enfant est évalué considérant les stratégies que l'enfant pense employer pour réagir.

Quelles stratégies et combien de stratégies il/elle dit vouloir employer pour résoudre le problème posé par la situation? 
37 A titre d'exemple, si un enfant dit qu'il va changer de langue, la démarche est acceptée comme stratégique. La stratégie que l'on appelle l'évitement, si elle implique une renonciation à l'échange, n'est pas considérée comme stratégique dans notre corpus. Ce choix épistémique est corroboré par le sentiment de tristesse dont les enfants font part et qui accompagne généralement la renonciation à l'échange (par exemple 'je suis triste', 'ça me donne mal', 'I feel bad', 'je vais pleurer', 'je rentre à la maison'...).

Neuf stratégies ont été retenues parmi l'ensemble des stratégies repérées chez des apprenants adultes et dépendant de quatre grandes catégories : métacognitives, affectives, socio-interactives et cognitives, définies par Oxford, (2008: 52). Plusieurs classifications ont été considérées pour opérer notre choix. La catégorisation d'Oxford étant la plus récente, nous avons choisi de la présenter compte tenu d'un travail de maturation des concepts déjà proposés trente ans auparavant (voir par exemple, Faerch et Kasper, 1983 ; ou Tarone, 1983). Parmi les neuf stratégies retenues, des critères précis ont été attribués de manière à éviter l'ambiguïté en ce qui concerne le classement.

Tableau 3 : Dénominations et définitions des stratégies qui ont permis la classification des réponses.

Attention dirigée: L'enfant se concentre sur un indice sémiotique tout en identifiant le problème. Il/elle essaie de s'ajuster.

Contrôle des émotions: l'enfant montre de la détermination pour communiquer et ne manifeste pas de sentiments négatifs.

Anticipation: L'enfant élabore un plan d'action tout en pensant à haute voix.

Mime: L'enfant propose de montrer quelque chose en mimant.

Coopération: L'enfant propose une action explicite pour communiquer avec l'autre enfant.

Changement de code: L'enfant propose une autre langue pour communiquer (y compris le langage des signes).

Imitation: L'enfant propose de faire la même chose que l'autre.

Clarification: L'enfant essaie d'obtenir des informations supplémentaires du chercheur.

Demande d'assistance: L'enfant réfère à une aide extérieure pour lui venir en aide.

\section{Méthodes statistiques}

Toutes les analyses ont été faites avec SPSS version 11.5. Toutes les valeurs sont significatives (test-bilatéral). Les comparaisons des intentions de communication ont été faites à l'aide d'un test $t$.

Pour comparer l'utilisation des stratégies par les monolingues versus multilingues ou LLE versus nLLE, nous avons utilisé un test d'analyse de variances à mesures répétées avec les groupes (monolingues versus multilingues et LLE versus nLLE), et avec les neuf stratégies 
en tant que variable à l'intérieur des groupes (monolingues versus multilingues et LLE versus nLLE).

Les données des différents pays ont été analysées indépendamment. Les résultats des analyses ne différant pas essentiellement, en termes d'âge, de sexe et d'utilisation des stratégies, les données des trois pays ont été réunies. Au total, les réponses de 101 participants sont incluses dans les analyses finales.

Dans la section suivante, nous analysons quelques réponses des enfants présentées cidessus pour illustrer la classification opérée. Ensuite, nous présentons les résultats statistiques.

\section{Résultats}

\section{Analyses des réponses des enfants LLE}

Dans l'exemple 1, ('I don't know. Hello. Je fais le langage des signes.'), Isabella commence par réfléchir. Cette réflexion est marquée par l'hésitation et la déclaration : 'Je ne sais pas.' Puis elle propose de 'faire le langage des signes' classifié comme un changement de code. On voit aussi qu'elle n'hésite pas au cours de l'entretien à changer la langue dans laquelle elle s'exprime. Ce recours au changement de code en tant que stratégie de communication se retrouve dans les réponses de beaucoup d'enfants soit comme Isabella pour mener à bien la conversation avec le chercheur, soit en tant que stratégie pour résoudre la tâche proposée. La réponse de Rob, 6 ans et 5 mois au moment de l'entretien (exemple 11) illustre bien ce phénomène: 'Je vais lui demander quelle langue il parle en français. Je lui dis les mots que je connais. Je demande à ma mère. J'espère qu'il comprend. Je lui dis : 'bouge pas!' Je fais le langage des signes.'

Rob s'appuie d'abord sur la langue qu'il connaît (attention dirigée). Il anticipe en élaborant un plan d'action qui n'est pas unilatéral (anticipation): il précise qu'il lui demande 'en français' et compte sur la coopération de son interlocuteur potentiel (coopération). Pour lui, la réponse de son interlocuteur n'est pas a priori problématique : il connait certainement certains mots 'Je lui dis les mots que je connais' (changement de code). Ce qui est frappant dans la réponse de cet enfant, c'est que la langue n'est pas envisagée du point de vue du sens qu'elle véhicule mais du point de vue de la rencontre. Le fait de dire des mots connus engage la bonne volonté de l'enfant à investir le territoire de l'autre, à en faire son ami.

Rob est monolingue, ses parents étant tous les deux francophones. Il vit à Zurich. Il est en apprentissage de l'allemand à l'école et pendant l'entretien, il a déclaré connaître quelques mots d'anglais et quelques mots d'espagnol. Il a ensuite recours à la demande d'assistance, auprès de sa maman probablement pour s'informer sur la langue en question si l'on en croit le déroulement de l'interaction, 'Je demande à ma mère'. Pour lui la langue est représentée par 'des mots'. 'J'espère qu'il comprend.' Cette simple déclaration est significative de tout le contenu affectif déployé lors de l'utilisation de stratégies de communication. Il montre combien l'enjeu de la communication exolingue peut-être important pour ces enfants. Du point de vue de la classification énoncée plus haut, cet enfant gère ses émotions de manière positive et efficace (gestion des émotions). L'échec d'une stratégie ne l'empêche pas de rebondir vers une autre, bien au contraire. La stratégie suivante contient un désir de coopération fort. 'Je lui dis : bouge pas!'. Cet enfant 
encourage son éventuel interlocuteur à ne pas abandonner, à ne pas s'en aller, à ne pas renoncer. L'ordre de ne pas bouger ressemble à une injonction du type : 'Ne pars pas, je ne suis pas au bout de mes ressources.' Et il continue en expliquant qu'il passe au langage des signes, stratégie dont l'efficacité est directe. Rob est donc d'un bout à l'autre de l'entretien décidé à communiquer. Malgré le fait que la situation soit imaginaire, l'enjeu lui semble d'importance et la langue n'est pas une barrière suffisante pour l'en empêcher.

\section{Analyse des réponses des enfants nLLE versus LLE}

46 A l'inverse, les réponses de Emma et Bryan, tous deux âgés de 6 ans et scolarisés dans la même école, bilingues en anglais et en français, sont éloquentes d'un point de vue du refus de la communication. 'Je suis pas contente. Je vais pas jouer parce que je comprends pas la langue.' Emma, en exprimant son désaccord, ('je suis pas contente'), révèle que l'enjeu pour elle n'est pas moins fort que pour Rob ou Isabella auparavant. Ce qui diffère, c'est sa manière de gérer la situation. Elle renonce à jouer à cause de la langue, ou plutôt, à cause de l'absence de langue commune. La langue est pour elle, obstacle à la communication. Bryan lui aussi propose dès l'entrée de jeu d'éviter l'enfant en question : 'Trouver une autre personne; jouer à la maison.' Il renonce à la communication en se repliant finalement dans un territoire linguistique stable: la maison.

Alicia a 5 ans. Elle répond: 'She speaks Italian? She speaks anything? French? I don't know. I do nothing. I don't play with her.' Par la stratégie de clarification, elle commence par s'informer sur la langue parlée par l'enfant. Elle dirige son attention sur le langage. Elle propose de changer de code en nommant le français qu'elle connaît ou l'italien. 'She speaks Italian? She speaks anything? French?' Sans succès, elle hésite 'I don't know' et renonce: 'I do nothing. I don't play with her.'

En revanche, Louis, bilingue en anglais et en français mais LLE puisqu'il apprend l'allemand à l'école, répond qu'il va 'Parler comme Deutch. Je parle une autre langue. Je vais parler moitié suisse. Etre gentil, l'aider, jouer si il est triste avec lui.' Il faut noter que les stratégies de Louis ne sont pas différentes de celles d'Alicia, sauf que Louis ne se contente pas de s'informer, il s'implique dans l'interaction. 'Je parle une autre langue.' On peut sourire en pensant à ce que 'moitié suisse' signifie pour lui. Vivant à Zurich, il est peut-être conscient de la parenté de l'allemand classique et du suisse-allemand. Il s'imagine donc probablement, tâchant de baragouiner en suisse allemand, à partir de ses ressources en allemand classique. Louis ne se contente pas du changement de code, et il n'est pas arrêté par la langue. Pour lui, les langues ne sont pas un obstacle à la communication mais un outil de communication. Si la langue lui semble être un recours évident, il ne s'y arrête pas. Il se met à la disposition de l'autre, il propose d' 'être gentil, l'aider, jouer si il est triste avec lui'. Il montre donc un contrôle de ses émotions assez remarquable et son projet marque encore une fois l'importance de cette situation pour lui. Il se met à la place de l'autre qui pourrait être attristé de ne pouvoir communiquer. C'est une démarche entièrement tournée vers l'échange humain plus que vers l'échange de paroles. La langue le soutient dans sa démarche. 


\section{Résultats statistiques}

\section{Volonté de communiquer}

Lorsque l'on compare les enfants monolingues ( $n=44)$ aux enfants bilingues ( $n=57)$, on trouve que $77 \%$ of des enfants monolingues cherchent à communiquer contre $66 \%$ des enfants bilingues. Cette différence n'est pas significative $(\chi 2=1.09 ; d f=1 ; p<0.5)$.

$\mathrm{Au}$ contraire lorsque l'on compare les enfants LLE ( $\mathrm{n}=72)$ aux participants nLLE ( $\mathrm{n}=29)$, la différence est significative; $79 \%$ des enfants LLE cherchent à communiquer contre $52 \%$ des enfants nLLE $(\chi 2=7.61 ; d f=1 ; p<0.01)$.

\section{Stratégies envisagées}

Si l'on compare les enfants monolingues aux enfants bilingues, on ne trouve pas de différence en ce qui concerne l'utilisation des stratégies ( $\mathrm{F}=0.93 ; d f=1,99 ; p=0.47$ ). L'interaction entre le type de stratégies et les groupes respectifs est, quant à elle, significative $(\mathrm{F}=2.12 ; d f=8,79 ; p<0.05)$. Cette dernière constatation est due à l'utilisation plus importante que font les enfants monolingues de l'anticipation (73\%) que les enfants bilingues (47\%). Pour toutes les autres stratégies la différence n'est pas significative.

Ces résultats apparaissent que l'on prenne en compte ou non l'âge des participants.

En ce qui concerne l'utilisation des stratégies pour les groupes (LLE et nLLE) les résultats montrent une utilisation des stratégies plus diversifiée et plus importantes des stratégies par les enfants LLE que par les enfants nLLE.

Les différences entre les moyennes d'utilisation des stratégies par les enfants LLE et nLLE sont significatives $(F=8.06 ; d f=1,99 ; p<0.01)$, de même que l'interaction entre stratégies et groupes (LLE versus nLLE) $F=2.67 ; d f=8.79 ; p<0.05$ ).

Cependant on trouve des différences importantes entre les deux groupes en fonction des stratégies utilisées. En effet, l'anticipation, l'attention dirigée, l'imitation et la demande d'assistance, présentent des différences significatives entre les deux groupes $(F=14.04 ; d f$ $=8,792 ; p<0.001$ ). À nouveau, ces résultats apparaissent que l'on inclue ou non l'âge des participants. D'autre part, la comparaison des résultats en fonction des pays ne fait pas apparaître de différence.

\section{Discussion}

\section{Nature de la compétence de communication}

La manière dont les enfants envisagent la communication dans des situations de communication exolingues est un facteur crucial qui affecte leur intention de participer à l'échange. Comme le montre la présente recherche, des expériences antérieures de d'apprentissage de langue(s) peuvent déterminer de manière significative le développement de ces deux éléments de la compétence de communication.

Ce résultat confirme l'idée selon laquelle la compétence de communication est dynamique, influencée par des expériences linguistiques et sociales préalables. De cette façon, on peut envisager le fait que LLE contribue à la construction d'une identité 
plurilingue positive mettant à la disposition de l'enfant un répertoire de stratégies plus varié pour mener à bien la communication et une intention de communiquer plus stable. Ainsi, l'observation des réactions des enfants face à ces situations donne le sentiment d'assister à l'émergence de la compétence de communication.

En effet, LLE semble stimuler la reconsidération de ces situations en permettant aux enfants de construire un répertoire d'expériences positives d'exolinguisme. De ce fait, LLE en développant la compétence stratégique, renforce la construction d'une identité plurilingue positive.

\section{Nature de LLE}

Le concept de LLE a été conçu sur la base du rôle hypothétique joué par l'expérience d'apprentissage d'une langue nouvelle en tant qu'expérience consciente par rapport à l'acquisition implicite d'une ou de deux langues.

61 Pour mener à bien cette étude, le concept de LLE a été opérationnalisé sur la base de trois critères. (1) l'expérience d'apprentissage d'une langue étrangère (2) dans un contexte d'apprentissage scolaire, (3) après l'âge de quatre ans. Cela implique que tous les enfants nLLE sont des enfants bilingues sans expérience d'apprentissage d'une langue étrangère à l'école. En revanche les enfants LLE peuvent être soit bilingues soit monolingues mais tous avec une expérience d'apprentissage d'une langue nouvelle. On remarque donc que le contexte formel et l'âge minimal d'exposition étant deux conditions à l'attribution d'une LLE, la recherche présentée ici ne répond pas à la question de savoir laquelle de ces deux variables est la plus importante en ce qui concerne l'effet obtenu. Cependant les caractéristiques suivantes peuvent être attribuées à LLE :

- L'effet de LLE dépasse certaines composantes du micro-contexte telles que la méthode d'apprentissage ou de l'enseignant. Tous les enfants fréquentaient les mêmes classes dans chacun des trois pays ce qui, bien entendu, n'implique pas l'homogénéité des expériences linguistiques vécues. Pourtant, on retrouve l'écart observé entre les enfants LLE et nLLE pour chacune des trois écoles et cette différence est constante avec l'âge. Les mêmes différences de réactions entre les enfants LLE et nLLE ont été observées en moyenne à tous les âges et dans chacun des trois pays.

- L'effet de LLE dépasse la dichotomie langue étrangère/ langue seconde. En effet, de manière à réduire les effets de différences potentielles entre les différents aspects contextuels (Porquier et Py, 2004), les enquêtes ont été limitées à un contexte éducationnel particulier, à savoir les écoles Françaises (internationales) à l'étranger. Le terme 'international' implique qu'au moins une langue de l'école n'est pas la langue de l'environnement. En revanche, c'est l'une des langues essentielles de l'école, (le plus souvent le français). Pour cette raison, la distinction 'étrangère' ou 'seconde' n'est pas essentielle dans le contexte des écoles internationales, l'apprentissage de ces langues est une question de survie dans ces écoles.

- L'effet de LLE dépasse l'idée d'une approche positive des langues qui serait dispensée dans le cadre des écoles internationales. En effet, quotidiennement confrontés à des langues et des cultures différentes, il est important de considérer le fait que les enfants qui fréquentent ces écoles pourraient avoir été plus désireux de communiquer dans ce contexte d'apprentissage particulier. Pourtant, si ce contexte positif d'enseignement peut avoir affecté positivement leurs performances, les deux groupes d'enfants LLE et nLLE ont bénéficié de cette approche. la différence entre LLE et nLLE est maintenue malgré un contexte positif d'apprentissage. LLE est donc un facteur qui dépasse aussi cet aspect du micro-contexte. 
Bien entendu, il est possible d'envisager l'influence d'autres facteurs sur le comportement des enfants face aux situations de communication exolingues. Cependant, comme nous l'avons vu précédemment, seules les variables qui affecteraient l'un ou l'autre groupe (LLE versus nLLE) différemment doivent être prises en compte. Nous pouvons donc conclure que LLE constitue un facteur d'influence majeur en ce qui concerne le traitement de la situation de communication exolingue.

\section{Implications pour la recherche}

Comme l'indique les résultats de cette recherche, l'une des méthodes envisageables pour mieux saisir la complexité de l'hétérogénéité des groupes plurilingues est de mieux cerner les effets des différents facteurs qui influencent la construction de la conscience métacognitive des enfants. L'approche par les biographies langagières permet d'aborder cette question de manière qualitative sans exclure une approche plus quantitative. Les résultats de l'étude présente tendent à montrer une grande hétérogénéité des groupes plurilingues tout en mettant en exergue l'effet majeur de l'expérience d'apprentissage d'une langue nouvelle (LLE). Ils montrent que LLE peut contribuer à l'émergence de certains aspects de la conscience métacognitive, tels que la conscience métacommunicative, qui en retour peuvent faciliter le traitement et l'issue des situations de communication exolingue.

\section{Implications pour la didactique des langues}

64 L'une des différences essentielles entre le contexte formel d'apprentissage et le contexte informel d'acquisition lorsque l'on apprend une langue nouvelle, peut être l'émergence d'une réflexion accompagnée, à propos de l'exposition aux situations de communication exolingue possibles (Porquier \& Py, 2006). Les étapes qui jalonnent le traitement des situations d'interaction exolingue en classe de langue incluent (1) l'explication de la situation à l'apprenant en terme de réactions appropriées ou non à la situation, (2) la planification de l'intervention et les différentes stratégies que l'on peut considérer pour la résoudre, (3) la confrontation des réactions de l'apprenant à la situation de communication exolingue. On peut alors spéculer que la réflexion engagée par l'enseignant sur l'interaction et sur le langage lui-même peut augmenter la volonté de communiquer et l'utilisation des stratégies. Ceci suggère que le développement de la compétence de communication pourrait bénéficier de stimulations concernant d'autres tâches que celle développées pour les besoins de l'étude présente. Par exemple, on pourrait envisager d'observer les réactions stratégiques d'enfants plurilingues face à d'autres situations de communication exolingue dans un contexte scolaire. Cela nous permettrait de mieux diriger les enseignants vers des solutions stratégiques des situations exolingue de classe.

\section{Limitations}

Que nous enseignent les réactions des enfants à des situations de communication imaginaire par rapport à leurs réactions éventuelles face à des situations de communication réelles? En 1998, Wenden répondait partiellement à cette question en 
affirmant que les stratégies que les participants pensent utiliser constituent une indication évidente de leur connaissance stratégique.

$$
\text { les enfants se construisent, peuvent être profondément influencées par le contexte }
$$
d'apprentissage éducationnel (Pintrich \& Groot, 1990). De la même manière, on peut envisager l'idée que LLE permet aux enfants d'assimiler les fondements d'un savoir-faire communicationnel qui pose la langue comme un objet, un outil de communication, et permet à l'enfant de se distancier par rapport à cet objet langue. Les résultats de l'étude présente placent l'étude des stratégies dans la sphère socioculturelle (Oxford et Schramm, 2008). De ce fait, ils stipulent une approche théorique du contexte qui articule les dimensions sociocognitives et une approche de l'interaction dynamique qui tiennent compte des situations, des acteurs et de ce que Mondada et Pekarek appellent 'leurs itinéraires sociaux' (2005).

71 Les résultats de la présente étude ont montré que LLE est un facteur majeur en ce qui concerne le développement de la conscience métacommunicative des enfants plurilingues.

1. La volonté de communiquer et la compétence stratégiques sont positivement affectées par LLE.

2. Les résultats des deux analyses, soit la volonté de communiquer et la compétence stratégique montrent que la compétence métacommunicative dépend plus de LLE ('expérience consciente d'apprentissage d'une langue étrangère après l'âge de quatre ans) que du bilinguisme en soi.

LLE ainsi défini, la communication exolingue est présentée à travers LLE, comme une opportunité pour la construction de l'identité plurilingue des enfants au contact de plus d'une langue (Le Pichon, De Swart, Ceginskas, van den Bergh, 2009). Enfin, nous pouvons conclure avec Porquier et Py à la présence d'une compétence exolingue qui 'fait partie de 
l'expérience sociale et linguistique des participants' et qui 'joue un rôle crucial dans l'appropriation', (Porquier \& Py, 2006 : 29).

\section{BIBLIOGRAPHIE}

Alber, J.L., \& Py, B. (1985). Interlangue et conversation exolingue. Cahiers du département des sciences du langage, Université de Lausanne.

Baird, J. R. (1990). Metacognition, purposeful inquiry and conceptual change. In E. Hegarty- Hazel (Ed.) The student laboratory and the science curriculum. London : Routledge.

Beacco, J.-C. (2007). L'approche par compétences dans l'enseignement des langues. Paris: Didier.

Benson, C. (2009). Designing effective schooling in multilingual contexts: going beyond bilingual models. In T. Skutnabb-Kangas, R. Phillipson, A. Mohanty, \& M. Panda (Eds.) Social justice through multilingual education. Multilingual Matters. 63-84.

Bialystok E. (2009). L'acquisition d'une deuxième langue, le bilinguisme pendant la petite enfance et leur impact sur le développement cognitif précoce. Ed rev. In: Tremblay RE, Barr RG, Peters $\mathrm{RDeV}$, eds. Encyclopédie sur le développement des jeunes enfants. Montréal, Québec : Centre d'excellence pour le développement des jeunes enfants; 1 -5. Disponible sur le site: http:// www.enfant-encyclopedie.com/documents/BialystokFRxp_rev.pdf. Page consultée le [09-09-2009].

Bialystok, E. (2001). Metalinguistic aspects of bilingual processing. Annual Review of Applied Linguistics, 21, 169-181. Cambridge University Press.

Biggs, J. B., \& Moore, P. J. (1993). The Process of Learning. New York: Prentice Hall.

Brown, A.L., \& Day, J.D. (1983). Macrorules for summarizing texts: The development of expertise. Journal of Verbal Learning and Verbal Behavior, 22, 1, 1-14.

Brown, A. L., Bransford, J. D., Ferrara, R. A., \& Campione, J. C. (1983). Learning, remembering, and understanding. In J. H. Flavell \& E. M. Markman (Eds.), Handbook of child psychology: 3. Cognitive development, 77-166. New York: Wiley.

Bruck, M., \& Genesee, F. (1995). Phonological Awareness in Young Second Language Learners. Journal of Child Language, 22, 2, 307-24.

Campbell, R., \& Sais, E. (1995). Accelerated metalinguistic (phonological) awareness in bilingual children. British Journal of Developmental Psychology, 13, 61-68.

Castellotti, V. (2008). L'école française et les langues des enfants : quelle mobilisation de parcours plurilingues et pluriculturels ? In J.L. Chiss (Dir.). Immigration, école et didactique du français, Paris, Didier, collection langues et didactique. 6, 1-25.

Cenoz, J., \& Jessner, U. (2000). English in Europe: The acquisition of a third language. Clevedon: Multilingual Matters.

Chamot, A. U., \& El-Dinary, P. B. (1999). Children's learning strategies in immersion classrooms. The Modern Language Journal, 83, 3, 319-341. 
Cole, M. (1995). Cultural-historical psychology : a meso-genetic approach. In L. M. W. Martin, K. Nelson \& E. Tobach (Eds.), Sociocultural psychology. Theory and practice of doing and knowing. 168-204, Cambridge University Press, Cambridge.

Cosnier, J. (1991). De l'amour du texte à l'amour du contexte. In, Fivaz-Depeursinge (dir.), Texte et contexte dans la communication. Cahiers critiques de thérapie familiale et de pratiques de réseaux, 13, 29-40. http://icar.univ-lyon2.fr/membres/jcosnier/articles/II-7_De_l'amour_du_texte.pdf

Coste, D., Moore, D. \& Zarate, G. (1997). Compétence plurilingue et pluriculturelle. Strasbourg: Conseil de l'Europe. Reprinted in Le Français dans le monde. Recherches et applications, 1998.

Cummins J. (1979). Linguistic interdependence and the educational development of bilingual children. Review of Educational Research, 49, 2, 222-251.

Dörnyei, Z., \& Scott, M. L. (1997). Communication strategies in a second language: Definitions and taxonomies. Language Learning, 47, 173-210.

Faerch, C. \& Kasper, G. (1983). Strategies in interlanguage communication. London: Longman.

Flavell, J. (2004). Theory-of-Mind Development: Retrospect and Prospect. Merrill-Palmer Quarterly, $50,3,274-290$.

Genesee, F., \& Nicoladis, E. (2006). Bilingual acquisition. In E. Hoff \& M. Shatz (Eds.), Handbook of language development, 324-342. Oxford: Blackwell.

Genesee, F., Tucker, G.R., \& Lambert, W.E. (1975). Communication skills of bilingual children. Child Development, 46, 1010-1014.

Georghiades, P. (2004). From the general to the situated: three decades of metacognition. International Journal of Science Education, 26, 3, 365-383.

Goldstein, F. C., \& Levin, H. S. (1987). Disorders of reasoning and problem-solving ability. In M. J. Meier, A. Benton, \& L. Diller (Eds.), Neuropsychological rehabilitation 327-354. New York: Guilford Press.

Grosjean, (1997). The bilingual individual. Interpreting: International journal of research and practive in interpreting, 2, 1/ 2, 163-87.

Gumperz, J. J. (1982). Discourse strategies. Cambridge: Cambridge University Press.

Hoffmann, C. (2001). Toward a description of trilingual competence. International Journal of Bilingualism, 5, 1, 1-17.

Houwer, de A. (1995). Bilingual language acquisition. In P. Fletcher \& B. MacWhinney (Eds.), The Handbook of Child Language. Oxford: Blackwell, 219-250.

Hurd, S., \& Lewis, T. (Eds.)(2008). Language learning in independent settings. Multilingual Matters. Jessner, U. (1997). Towards a dynamic view of multilingualism. In M. Putz (ed.) Language Choices? Conditions, Constraints and Consequences, 17-30. Amsterdam: Benjamins.

Jorda, M.P.S. (2005). Pragmatic production of third language learners of English: A Focus on request acts modifiers. International Journal of Multilingualism, 2, 2, 84-104.

Krashen, S.D. (1981). Second language acquisition and second language learning. New-York: Pergamon Press.

Le Pichon, E., De Swart, H., Ceginskas, V., van den Bergh, H. (2009). Language learning experience in school context and metacognitive awareness of multilingual children. International Journal of Multilingualism, 1-23. 
Le Pichon, E., Vorstman, J.A.S., De Swart H., \& Van Den Bergh H., (2009). Effect of learning a new language on the willingness to communicate. Toegepaste Taalwetenschap in Artikelen, 81, 31-40.

Lee Swanson, H. (1990). Influence of Metacognitive Knowledge and Aptitude on Problem Solving. Journal of Educational Psychology, 82, 2, 306-314.

McIntyre, P.D., Clément, R., Dörnyei, Z., \& Noels, K. (1998). Conceptualizing willingness to communicate in a L2: A situational model of L2 confidence and affiliation. Modern Language Journal, 82, 545-562.

Mondada, L., \& Pekarek, S. (2005). Interaction sociale et cognition située : quels modèles pour la recherche sur l'acquisition des langues? Acquisition et Interaction en Langue Étrangère [En ligne], Approches interactionnistes de l'acquisition des langues étrangères. http://aile.revues.org/ document947.html.

Moore, D. \& Castellotti, V. (2008). Perspectives de la recherche francophone autour de la notion de compétence plurilingue. In D. Moore et V. Castellotti. La compétence plurilingue, Regards francophones, 11-24. Berne : Transversales, Peter Lang.

Myers-Scotton, C. (2006). Multiple Voices. An Introduction to Bilingualism. Blackwell Publishing. Narcy-Combes, J-P., Tardieu, C., Le Bihan, J.C., Aden,J., Delasalle, D., Larreya, P., Raby, F. (2008). L'anglais à l'école élémentaire. Les Langues Modernes, 4, 1-11. www.aplv-languesmodernes.org Noyau, C. (1998). Temporalité et récit dans l'acquisition du langage en situation bilingue. LINX, 38, 7-18.

Oxford, R. \& Schramm, K. (2007). Bridging the gap between psychological and sociocultural perspectives on L2 Learner Strategies. In A. Cohen \& E. Macaro, (Eds.) Language Learner Strategies. Oxford: Oxford University Press.

OXFORD, R. (2008) Hero with a thousand faces: Learner Autonomy, learning strategies and learning tactics in independent language learning. In Stella Hurd \& Tim Lewis (Eds.) Language learning in independent settings. Multilingual Matters. 41-66.

Oxford, R.L. 2003. 'Language Learning styles and strategies: concepts and relationships.' International Review of Applied Linguistics in Language Teaching, 41, 271-278.

Pavlenko, A. \& Blackledge, A. (2004). Introduction: new theoretical approaches to the study of negotiation of identities in multilingual contexts. In A. Pavlenko \& A. Blackledge (eds.). Negotiation of identities in multilingual contexts. 1-33. Multilingual Matters.

Pintrich, P.R. \& De Groot E. (1990). Motivational and self-regulated learning components of classroom academic performance. Journal of Educational Psychology, 82, 1, 33-50.

Porquier, R. \& Py, B. (2004). Apprentissage d'une langue étrangère : contexte et discours. Credif: Essais, Didier.

Porquier, R. (1979). Stratégies de communication en langue non-maternelle. Travaux du centre de recherches sémiologiques, Neuchâtel, Université, 39-52.

Rubin, H., \& Turner, A. (1989). Linguistic awareness skills in grade one children in a French immersion setting. Reading and writing, 1, 73-86.

Schneider, W., \& Pressley, M. (1997). Memory development between two and twenty. Mahwah, NJ: Lawrence Erlbaum Associates.

Tarone, E., (1983). Teaching Strategic Competence in the Foreign Language Classroom. In: Savignon, Sandra J., Ed. and Berns, Margie S., Ed. Communicative Language Teaching: Where Are We 
Going? Urbana, Language Learning Laboratory, University of Illinois at Urbana-Champaign, 1983; see FL 016 358. 1-11. Disponible sur: http://www.eric.ed.gov/

Wenden, A. (1998). Metacognitive knowledge and language learning. Applied Linguistics, 19, 4, 515-537.

YELLAND, G.W., POLLARD, J., \& MERCURI, A. (1993). The metalinguistic benefits of limited contact with a second language. Applied Psycholinguistics, 14, 423-444.

Zeidner, M., \& Matthews, G. (2000). Personality and intelligence. In R.J. Sternberg (Ed.), Handbook of human intelligence (2nd ed., 581-610). Cambridge University Press.

\section{NOTES}

1. Les prénoms des enfants ont tous été modifiés afin de respecter leur anonymat.

\section{RÉSUMÉS}

De nombreux facteurs de nature socioculturelle, tels que les contextes d'apprentissage ou d'acquisition de la ou des langues, contribuent à la construction de répertoires plurilingues. Cependant, il reste encore difficile d'identifier précisément quels sont ces facteurs et quel est leur rôle potentiel dans le développement de l'enfant plurilingue (Mondada \& Pekarek, 2005; Porquier \& Py, 2004).

La présente recherche explore cette question en contrastant deux contextes acquisitionnels.

L'hypothèse a été posée selon laquelle le niveau de conscience métacommunicative des enfants plurilingues dépend plus du contexte d'apprentissage d'une langue étrangère que du bilinguisme en soi.

L'évaluation des représentations métacommunicationnelles de 101 enfants plurilingues exposés à des situations de communication exolingues radicales et imaginaires montre que le contexte d'apprentissage/ acquisition a des conséquences majeures, particulièrement sur les intentions de communication et sur la compétence stratégique des enfants plurilingues.

\section{AUTEUR}

\section{EMMANUELLE LE PICHON - VORSTMAN}

Université d'Utrecht, Pays-Bas et Université François-Rabelais de Tours 